\title{
Barriers to and facilitators for use of augmentative and alternative communication and voice restorative devices in the adult intensive care unit: a scoping review protocol
}

\author{
Laura Istanboulian ${ }^{1 *}$ (D), Louise Rose ${ }^{2}$, Yana Yunusova ${ }^{3}$, Franklin Gorospe ${ }^{1}$ and Craig Dale ${ }^{1}$
}

\begin{abstract}
Background: Mechanically ventilated patients in the intensive care unit (ICU) experience profound communication impairment, placing them at risk for poor physical and psychological outcomes. Patient communication strategies such as augmentative and alternative communication (AAC) and voice restorative devices are recommended to facilitate communication. These strategies, however, are inconsistently adopted in ICU practice signaling utilization barriers. Our objective is to map and synthesize the current evidence-base for stakeholder-reported barriers and facilitators to patient communication strategy utilization for adults with an advanced airway in the ICU.

Methods and analysis: We will use Arskey and O'Malley's recommended methods to conduct a scoping review using a rapid review framework to streamline the process. A single reviewer will conduct a search and an initial screen of titles and abstracts from five electronic databases (MEDLINE, EMBASE, the Cochrane Library, Cumulative Index to Nursing and Allied Health Literature [CINAHL], and PsychInfo) from 1990 to present to identify English language peer-reviewed studies. Subsequently, two reviewers will independently screen a shorter list of studies for inclusion. We will also search the reference lists of eligible studies. Two reviewers will independently extract study characteristics, communication strategy, and stakeholder reported barriers and facilitators. We will code and categorize the extracted barriers and facilitators according to the Theoretical Domains Framework (TDF), an integrative framework of behavior change.

Discussion: To our knowledge, this will be the first scoping review to map and synthesize reported barriers and facilitators to communication strategy utilization in the adult ICU using a theoretical framework. The results of this scoping review will help to identify trends and gaps in the current evidence-base and support recommendations for improving patient-centered practice, policy, and research related to successfully establishing ICU patient communication.
\end{abstract}

Keywords: Communication, Critical care, Augmentative and alternative communication, Barriers and facilitators, Theoretical Domains Framework

\footnotetext{
* Correspondence: Laura.Istanboulian@utoronto.ca

'Lawrence S. Bloomberg Faculty of Nursing, University of Toronto, 155

College Street, Suite 130, Toronto, ON M5T 1P8, Canada

Full list of author information is available at the end of the article
}

(c) The Author(s). 2019 Open Access This article is distributed under the terms of the Creative Commons Attribution 4.0 International License (http://creativecommons.org/licenses/by/4.0/), which permits unrestricted use, distribution, and reproduction in any medium, provided you give appropriate credit to the original author(s) and the source, provide a link to the Creative Commons license, and indicate if changes were made. The Creative Commons Public Domain Dedication waiver (http://creativecommons.org/publicdomain/zero/1.0/) applies to the data made available in this article, unless otherwise stated. 


\section{Background}

Treatment and monitoring of patients experiencing lifethreatening conditions occur in the intensive care unit (ICU) [1]. The core treatment modality in the ICU is mechanical ventilation through an advanced airway interface, including an oral endotracheal or a tracheostomy tube. For patients requiring mechanical ventilation, the placement of an advanced airway interrupts vocalization and contributes to profound communication impairment. The shifting treatment paradigm from full to light or no-sedation has made communication impairment an increasingly important patient safety priority since less sedation results in patients who are more awake and struggling to be understood [2-5]. One study of mechanically ventilated patients across six specialty ICUs in an American academic health system estimated that over $50 \%$ of ICU patients have sufficient alertness to communicate with the people around them but may not be understood due to communication impairment arising from an artificial airway [6].

Mechanically ventilated patients describe communication impairment as one of the most stressful, dehumanizing, and frustrating events of hospital admission [7-9]. Communication impairment places ICU patients at risk for physical and psychological harm as it negatively impacts communication of disease symptoms, treatment responses, as well as participation in decision-making [6, $8,10,11]$. Impaired patient communication is a modifiable risk factor for over- and under-recognition and treatment of symptoms of critical illness such as pain, anxiety, agitation, and dyspnea [6, 12]. Poor symptom management contributes to delirium, physical restraint use, and prolonged mechanical ventilation [13, 14]. In the long term, patient communication impairment also contributes to the development of chronic pain, depression, and post-traumatic stress disorder [10].

Patient communication in the ICU can be supported by strategies including augmentative and alternative communication (AAC) and voice restorative devices. AAC refers to all forms of communication, other than oral speech, that are used to express messages [15]. AAC can include "unaided" strategies (e.g., facial expressions, mouthing words, and gesturing) or "aided" strategies which include low-tech (e.g., alphabet or picture boards and writing instruments such as paper and pen) and high-tech (e.g., specialized computer communication interfaces) devices [15]. Phonation or voice restorative devices for patients with an advanced airway refer to voice enabling tracheostomy-based communication aids such as those that require cuff deflation (e.g., one-way speaking valves and ventilator-adjusted leak speech) and those that do not require cuff deflation (e.g., talking tracheostomy with dynamic cuff and fenestrated inner cannula) $[16,17]$. Two recent systematic reviews report aided
AAC strategies are effective in improving ICU patient satisfaction and reducing communication difficulty [18, 19]. Voice restorative devices are also demonstrated to enhance communication by restoring voice in the presence of an advanced airway [17].

Despite the range of available strategies and reported efficacy in reducing communication difficulties, patient communication strategies are inconsistently adopted in ICU practice [20-22]. Variable adoption of communication strategies in ICU practice signals the presence of utilization barriers [20-23]. A better understanding of the barriers to and facilitators for the utilization of patient communication strategies from the perspective of key stakeholders (e.g., patients, patient communication partners such as nurses, physicians, interdisciplinary clinicians, family members) can potentially improve patient communication strategy utilization in the adult ICU.

One recent systematic review reporting barriers to AAC use in the ICU only included experimental, quasiexperimental, and observational studies [18]. This review reported barriers to usage were device characteristics, the clinical condition of the patient, and constraints in staff time. The lack of inclusion of qualitative and mixed method approaches may inhibit understanding of key stakeholder perspectives and contextual issues relevant to the utilization of patient communication strategies in the ICU. Furthermore, previous reviews exploring barriers to patient communication in the adult ICU have not applied a theoretical framework to understand communication strategy utilization barriers and facilitators. The use of an implementation theory that includes behavioral and contextual determinants, such as the Theoretical Domains Framework (TDF), may help to better understand and identify potentially modifiable barriers to and facilitators for communication strategy utilization in the ICU $[24,25]$.

\section{Methods and design Aim and objectives}

The primary aim of this scoping review is to answer the question: What are stakeholder reported barriers to and facilitators for patient communication strategy utilization for adults with an advanced airway in the ICU? To answer this question, we will conduct a scoping review with the primary aim of mapping of the current evidence on barriers to and facilitators for patient communication strategy (including AAC and voice restorative device) utilization for adults with an advanced airway in the ICU. The secondary aim of this review is to use the TDF to better understand barriers to and facilitators for communication strategy utilization, highlight key trends and gaps, and to inform evidence-based patient-centered practice, policy, and research. To our knowledge, this will be the first 
scoping review to map and synthesize reported barriers to and facilitators for to communication strategy utilization in the adult ICU using a theoretical framework.

\section{Methods}

Our scoping framework will be informed by the scoping review methods suggested by Arskey and O'Malley, and advanced by Levac and Colquhoun [26-28]. We will follow the scoping review reporting methods outlined by the Preferred Reporting for Items for Systematic reviews and Meta-Analysis Protocols (PRISMA-P) (Additional file 1) [29]. We will also use rapid review methods to expedite the review process. A rapid review is a type of knowledge synthesis in which components of the systematic review process are simplified or omitted to produce information in a shorter period of time [30]. The rapid review components we will use include omission of gray literature and a single-reviewer system to perform the first screen of all titles and abstracts.

\section{Information sources}

The research team developed a comprehensive search strategy (Additional file 2) in consultation with a health sciences information specialist. Our search strategy will be used in Ovid Medline Epub Ahead of Print, InProcess and Other Non-Indexed Citations, Ovid Medline Daily and Ovid Medline, Ovid Embase, and translated to Ovid PsycINFO, EBSCO Cumulative Index to Nursing and Allied Health Literature (CINAHL), and the Cochrane Library.

Including a complete gray literature search can be time consuming with very minimal relevant results and questionable replicability [31]. Omitting the gray literature as part of a rapid evidence assessment introduces the risk of selection bias [30,31]. To mitigate the risk of missing relevant studies, we will hand search reference lists of included studies and recent systematic reviews and include those meeting inclusion criteria $[18,19,30]$.

\section{Eligibility criteria}

We will combine and deduplicate all electronic database searches in Endnote $^{\mathrm{Tm}}$ X9 [32]. One reviewer (LI) will perform an initial screen of titles and abstracts removing studies not meeting the eligibility criteria. The eligibility criteria are listed in Table 1. The remaining studies will be imported into Covidence ${ }^{\text {max }}$. Two independent reviewers (LI/FG) will screen study titles and abstracts against the eligibility criteria and subsequently screen full-text articles to aid in decision making about inclusion. Discrepancies will be resolved by discussion and a third reviewer (CD) as arbiter if necessary. Reference lists of included studies and recent systematic reviews will be screened by title and then full text by the process described above to decide about inclusion $[18,19]$.

We will include published studies from 1990 to present reporting quantitative, qualitative, mixed or multi-method designs, including both comparative (e.g., randomized, controlled, cohort, quasi-experimental) and non-comparative (e.g., survey, narrative, audit) methods. We will include all study designs to keep our search broad enough to capture diverse stakeholder reported barriers or facilitators of patient communication strategy utilization in the ICU. We will, however, exclude reviews, protocols, and opinion pieces including editorials and letters since these designs will likely not report our outcome of interest. For practical reasons, our search will be limited to English language studies. The 1990year limit aligns with the paradigm shift to minimal ICU sedation practices. We also consider studies published more than 25 years ago may not be relevant to current barriers and facilitators to patient communication strategy utilization in the ICU.

Table 1 Inclusion and exclusion criteria

\begin{tabular}{|c|c|c|}
\hline & Inclusion & Exclusion \\
\hline Population/setting & $\begin{array}{l}\text { - Adult (age 18+) ICU patients and their communication partners (i.e., } \\
\text { nurses, physicians, interdisciplinary clinicians, family members) } \\
\text { - ICU, specialized weaning centers, and high-dependency in patient } \\
\text { settings } \\
\text { - Patients with an advanced airway (oral endotracheal tube, } \\
\text { tracheostomy) }\end{array}$ & $\begin{array}{l}\text { - Emergency department, postoperative recovery units, } \\
\text { hospital floors/wards, psychiatry, long-term care, and } \\
\text { home settings }\end{array}$ \\
\hline Intervention & $\begin{array}{l}\text { - Patient communication strategies including AAC (unaided strategies, } \\
\text { aided strategies (low- and high- tech); voice restorative devices }\end{array}$ & \\
\hline Comparator & - Studies with comparison or no-comparison group will be included & \\
\hline Outcomes & $\begin{array}{l}\text { - Stakeholder (patients and communication partners including nurses, } \\
\text { physicians, interdisciplinary clinicians, family members)—reported } \\
\text { barriers and facilitators to patient communication strategies }\end{array}$ & $\begin{array}{l}\text { - Studies without stakeholder reported barriers or } \\
\text { facilitators to patient communication strategies }\end{array}$ \\
\hline Type of study & - All study designs & $\begin{array}{l}\text { - Editorials, letters, protocols, reviews, education pieces, } \\
\text { reports, working papers, government documents, white } \\
\text { papers, and evaluations }\end{array}$ \\
\hline
\end{tabular}




\section{Data extraction}

Two reviewers (LI/FG) will independently extract data using an iteratively developed data extraction form. Study identifiers and data to be extracted are listed in Table 2.

Stakeholder-reported outcomes will be extracted if they are reported as barriers or facilitators to patient communication strategy utilization. Stakeholders include patients and their communication partners (e.g., nurses, physicians, interdisciplinary clinicians, family members). We will define barriers as any physiological, psychological, cognitive, or contextual conditions reported to reduce or negatively affect patient communication strategy utilization in the adult ICU [33]. We will define facilitators as physiological, psychological, cognitive, or contextual conditions reported to enhance or positively affect patient communication strategy utilization in the adult ICU [33].

We anticipate the extraction of the data will be an iterative process that depends on the evidence found in our search [27]. We also anticipate that data extracted will vary based on the type of study and data presented [27]. For example, for qualitative studies, descriptions of individual barriers and facilitators will be extracted verbatim unless only reported in a synthesized format. For quantitative studies, reported outcomes will be extracted and categorized as barriers or facilitators according to the approach described by Weatherson et al. (2017) (i.e., if $50 \%$ or more participants identify ease of use of a communication device/strategy, "ease of use" will be categorized as a facilitator) [34]. We will note the reporting stakeholder source of each extracted barrier and facilitator.

To ensure reliability, two reviewers (LI/FG) will pilot the barrier and facilitator extraction process then meet to compare findings. They will repeat this process for 35 articles, or until reliability is reached and the extraction tool is adapted. Upon completing the extraction, the two reviewers will meet to determine agreement on the presence or absence of barriers and facilitators

Table 2 Study identifiers and data to be extracted

- First author and year of publication

- Full reference

- Location of publication

- Study design

- Participant (e.g., patient, nurse, physician, interdisciplinary clinician,

family) and unit characteristics

- Stated aim(s) of study

- Communication strategy (e.g., AAC unaided or aided, low or high tech, voice restorative device)

- Verbatim description of barriers and facilitators to communication strategy utilization with reporting stakeholder noted

- Stakeholder reported outcomes related to communication strategy utilization (e.g., ease of tool use; acceptability of tool; satisfaction; speech intelligibility)

$A A C$, augmentative and alternative communication within each paper. The authors will solve discrepancies through discussion, rereading source material and collaboration. In the event that agreement cannot be reached, the opinion of a third reviewer (CD) will determine the final result.

\section{Presentation of findings}

Following the PRIMSA-ScR scoping review extension guide, we will present study screening and inclusion in a PRISMA chart [35]. We will also present a summary table of the studies meeting eligibility criteria including the stated aims of the study, study design, study setting, participant characteristics, and patient communication strategy. We will present extracted and coded barriers and facilitators both quantitatively and qualitatively.

\section{Synthesis of barriers and facilitators}

Barriers and facilitators will be coded and categorized into the 14 domains of the TDF using a recommended coding approach $[25,36,37]$. A coding book will be developed by the research team prior to the coding process and iteratively modified to ensure accuracy, consistency, and transparency of the interpretive process of categorizing barriers and facilitators to the TDF domains [37]. Two reviewers will independently and deductively code the barriers and facilitators and will meet to discuss coding discrepancies until a final TDF categorization is accomplished. The resulting TDF categorization will be reviewed by the entire authorship team to determine if any alternative categorizations are plausible. We will then review the full list of extracted barriers and facilitators categorized each domain and determine the number of unique barriers and facilitators in each. Three criteria will be used to judge relevance of a TDF domain: (1) relatively high frequency of barriers or facilitators, (2) presence of conflicting barriers or facilitators, and (3) evidence of stakeholder beliefs that impact utilization of a communication strategy [25]. The authors will solve discrepancies through discussion, rereading source material, and collaboration.

We will present a quantitative summary of the barriers and facilitators to patient communication strategy utilization in the adult ICU including the frequency of reported barriers and facilitators in included studies (as counts within each TDF domain and proportions overall). We will also present the frequency (proportion) of barriers and facilitators in each TDF domain according to study design, communication strategy, and the stakeholder reporting the barrier or facilitator.

A qualitative analysis will be performed to provide major themes of barriers and facilitators of each TDF domain with reference to stakeholder and communication strategy [38]. Following an immersive reading of the coded TDF domains, we will look for recurring patterns 
in the data. Peer debriefing and reflexive writing will be used to bring meaning and coherence to barrier and facilitator concepts linking substantial portions of the data together. The overarching themes will represent patterns identified in the data comprising domain concepts most likely to influence communication behaviors including those that are potentially modifiable. Verbatim exemplars will be provided for the included TDF domains.

\section{Quality assessment}

We will appraise the risk of bias of included studies using the Mixed Methods Appraisal Tool (MMAT) Version $2018[39,40]$. The MMAT is an easy to use tool demonstrating moderate to perfect interrater reliability [41]. Two reviewers (LI/FG) will independently appraise study quality. Though we will not exclude studies of low quality, we will use the results to describe the rigor of the included studies.

\section{Discussion}

The primary aim of this scoping review is to answer the question: What are stakeholder reported barriers to and facilitators for patient communication strategy utilization for adults with an advanced airway in the ICU? To answer this question, we will map and synthesize stakeholder reported barriers and facilitators in the current peerreviewed evidence-base to the domains of the TDF. We will build upon existing reviews by incorporating the qualitative and mixed methods literature, which may offer new stakeholder perspectives and contextual understandings. Since the TDF is a broad framework about behavior change that includes individual and environmental/contextual domains, it is a suitable framework to categorize the potential wide range of stakeholder reported barriers to and facilitators of communication strategy utilization in the adult ICU.

As physical and psychological harm can result from communication impairment in adult ICU patients treated with an advanced airway, it is critical to address this complex issue. The synthesis of barriers and facilitators using the TDF will allow us to better understand and highlight potentially modifiable antecedents to behavior change related to communication strategy utilization in the adult ICU. Furthermore, using the TDF to categorize barriers and facilitators reported in the current evidence base provides a theoretical foundation for future interventions targeting behavior change. The results of this review will also help to identify trends and gaps in the current peer-reviewed evidence base and support recommendations for improving patientcentered practice and policy related to successfully establishing and sustaining adult patient communication in the ICU.

\section{Strengths and limitations}

The strengths of this scoping protocol include the use of a transparent and established scoping review methodology and reporting structure; a systematic search of five electronic databases developed in consultation with a health sciences information specialist; systematic screening and data extraction carried out by two independent reviewers for all steps except the initial screen; the inclusion of qualitative and mixed methods studies; the inclusion of a quality assessment step using the MMAT; and the use of a theoretical framework to map and synthesize barriers and facilitators to patient communication strategy utilization that spans multiple levels of influence in the adult ICU.

Limitations of our protocol include selection bias by restricting our search to publications in English after 1990, the use of a rapid review approach including omission of gray literature, and single reviewer first screen of titles and abstracts. To mitigate risk of selection bias introduced by our rapid evidence selection processes, we will follow recommended strategies to ensure replicability (methodological transparency), objectivity and accuracy (two independent screeners for second review of titles/abstracts and full text, two independent data extractors with a detailed process of reaching agreement, use of a quality assessment tool), and comprehensiveness (multiple databases and hand-searching of reference lists) $[42,43]$.

\section{Supplementary information}

Supplementary information accompanies this paper at https://doi.org/10. 1186/s13643-019-1232-0.

Additional file 1. PRISMA-P 2015 Checklist. Description of data: PRISMAP Checklist.

Additional file 2. Results of the pilot database search. Description of data: Results of search performed March 17, 2019 in Ovid MEDLINE: Epub Ahead of Print, In-Process \& Other Non-Indexed Citations, Ovid MEDLINE ${ }^{\circledR}$ Daily and Ovid MEDLINE ${ }^{\circledR}<1946$-Present.

\section{Abbreviations}

AAC: Augmentative and alternative communication; ICU: Intensive care unit; MMAT: Mixed Methods Appraisal Tool; TDF: Theoretical domains framework

\section{Acknowledgements}

The authors would like to thank Mikaela Gray, Liaison \& Education Librarian, Gerstein Science Information Centre, University of Toronto.

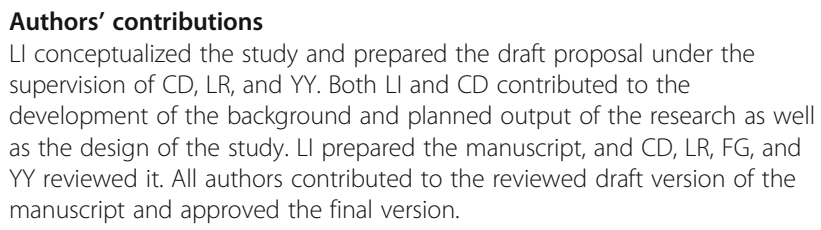


Innovation Development Grant from the Michael Garron Hospital, Toronto, Canada.

\section{Availability of data and materials}

All data generated or analyzed during this study will be included in the published scoping review article.

\section{Ethics approval and consent to participate}

Not applicable.

\section{Consent for publication}

Not applicable.

\section{Competing interests}

The authors declare that they have no competing interests.

\section{Author details}

'Lawrence S. Bloomberg Faculty of Nursing, University of Toronto, 155 College Street, Suite 130, Toronto, ON M5T 1P8, Canada. ${ }^{2}$ Florence Nightingale Faculty of Nursing, Midwifery and Palliative Care, King's College, 57 Waterloo Rd, Lambeth, London SE1 8WA, UK. ${ }^{3}$ Department of Speech and Language Pathology, University of Toronto, 160-500 University Ave., Toronto, ON M5G 1 V7, Canada.

Received: 17 May 2019 Accepted: 12 November 2019

Published online: 06 December 2019

\section{References}

1. Care in Canadian ICU's. Canadian Institute for Health Information. 2016.

2. Egerod I. Cultural changes in ICU sedation management. Qual Health Res. 2009;19(5):687-96.

3. Devlin JW, Pandharipande PP. Light sedation is the goal: making the evidence heavier. Crit Care Med. 2018;46(6):1003-4

4. Devlin JW, Skrobik Y, Gelinas C, Needham DM, Slooter AJC, Pandharipande PP, et al. Clinical practice guidelines for the prevention and management of pain, agitation/sedation, delirium, immobility, and sleep disruption in adult patients in the ICU. Crit Care Med. 2018;46(9):e825-e73.

5. Mehta S, Burry L, Cook D, Fergusson D, Steinberg M, Granton J, et al. Daily sedation interruption in mechanically ventilated critically ill patients cared for with a sedation protocol: a randomized controlled trial. JAMA. 2012; 308(19):1985-92

6. Happ MB, Seaman JB, Nilsen ML, Sciulli A, Tate JA, Saul M, et al. The number of mechanically ventilated ICU patients meeting communication criteria. Heart Lung. 2015;44(1):45-9.

7. Karlsson V, Lindahl B, Bergbom I. Patients' statements and experiences concerning receiving mechanical ventilation: a prospective video-recorded study. Nurs Inq. 2012;19(3):247-58.

8. Baumgarten M, Poulsen I. Patients' experiences of being mechanically ventilated in an ICU: a qualitative metasynthesis. Scand J Caring Sci. 2015;29: 205-14.

9. Guttormson $J$, Bremer $\mathrm{KL}$, Jones RM. "Not being able to talk was horrid": a descriptive, correlational study of communication during mechanical ventilation. Intensive Crit Care Nurs. 2015;31(3):179-86.

10. Khalaila R, Zbidat W, Anwar K, Bayya A, Linton DM, Sviri S. Communication difficulties and psychoemotional distress in patients receiving mechanical ventilation. Am J Crit Care. 2011;20(6):470-9.

11. Turnbull AE, Sahetya SK, Needham DM. Aligning critical care interventions with patient goals: a modified Delphi study. Heart Lung. 2016:45(6):517-24.

12. Radtke JV, Baumann BM, Garrett KL, Happ MB. Listening to the voiceless patient: case reports in assisted communication in the intensive care unit. J Palliat Med. 2011;14(6):791-5.

13. Barnes-Daly MA, Pun BT, Harmon LA, Byrum DG, Kumar VK, Devlin JW, et al. Improving health care for critically ill patients using an evidence-based collaborative approach to ABCDEF bundle dissemination and implementation. Worldviews Evid Based Nurs. 2018:15(3):206-16.

14. Chanques G, Jaber S, Barbotte E, Violet S, Sebbane M, Perrigault PF, et al. Impact of systematic evaluation of pain and agitation in an intensive care unit. Crit Care Med. 2006;34(6):1691-9.

15. Miglietta MA, Bochicchio G, Scalea TM. Computer-assisted communication for critically ill patients: a pilot study. J Trauma. 2004;57(3):488-93.
16. Morris LL, Bedon AM, McIntosh E, Whitmer A. Restoring speech to tracheostomy patients. Crit Care Nurse. 2015;35(6):13-27 quiz 8.

17. Freeman-Sanderson AL, Togher $L$, Elkins MR, Phipps PR. Return of voice for ventilated tracheostomy patients in ICU: a randomized controlled trial of early-targeted intervention. Crit Care Med. 2016;44:1075-81.

18. Carruthers $\mathrm{H}$, Astin $\mathrm{F}$, Munro W. Which alternative communication methods are effective for voiceless patients in Intensive Care Units? A systematic review. Intensive Crit Care Nurs. 2017:42:88-96.

19. Ten Hoorn S, Elbers PW, Girbes AR, Tuinman PR. Communicating with conscious and mechanically ventilated critically ill patients: a systematic review. Crit Care. 2016:20(1):333.

20. Happ MB, Garrett K, Thomas DD, Tate J, George E, Houze M, et al. Nurse patient communication interactions in the intensive care unit. Am J Crit Care. 2011;20(2):e28-40,

21. Freeman-Sanderson A, Morris K, Elkins M. Characteristics of patient communication and prevalence of communication difficulty in the intensive care unit: an observational study. Aust Crit Care. 2018.

22. Rose L, Fowler RA, Fan E, Fraser I, Leasa D, Mawdsley C, et al. Prolonged mechanical ventilation in Canadian intensive care units: a national survey. J Crit Care. 2015;30(1):25-31.

23. Mobasheri $\mathrm{MH}$, King $\mathrm{D}$, Judge $\mathrm{S}$, Arshad $\mathrm{F}$, Larsen $M$, Safarfashandi $Z$, et al. Communication aid requirements of intensive care unit patients with transient speech loss. Augment Altern Commun. 2016;32(4):261-71.

24. Michie S, Johnston M, Abraham C, Lawton R, Parker D, Walker A, et al. Making psychological theory useful for implementing evidence based practice: a consensus approach. Qual Saf Health Care. 2005;14(1):26-33.

25. Atkins L, Francis J, Islam R, O'Connor D, Patey A, Ivers N, et al. A guide to using the theoretical domains framework of behaviour change to investigate implementation problems. Implement Sci. 2017;12(1):77.

26. Levac D, Colquhoun $\mathrm{H}, \mathrm{O}$ 'Brien KK. Scoping studies: advancing the methodology. Implement Sci. 2010;5(69). https://doi.org/10.1186/1748-5908-5-69.

27. Arksey H, O'Malley L. Scoping studies: towards a methodological framework Int J Soc Res Methodol. 2005;8(1):19-32

28. Colquhoun HL, Levac D, O'Brien KK, Straus S, Tricco AC, Perrier L, et al. Scoping reviews: time for clarity in definition, methods, and reporting. J Clin Epidemiol. 2014:67(12):1291-4.

29. Shamseer L, Moher D, Clarke M, Ghersi D, Liberati A, Petticrew M, et al. Preferred reporting items for systematic review and meta-analysis protocols (PRISMA-P) 2015: elaboration and explanation. BMJ. 2015;350:g7647.

30. Ganann R, Ciliska D, Thomas H. Expediting systematic reviews: methods and implications of rapid reviews. Implement Sci. 2010;5:56.

31. Adams RJ, Smart P, Huff AS. Shades of grey: guidelines for working with the grey literature in systematic reviews for managment and organizational studies. Br Acad Manag. 2017;19:432-54.

32. Bramer WM, Giustini D, de Jonge GB, Holland L, Bekhuis T. De-duplication of database search results for systematic reviews in EndNote. J Med Libr Assoc 2016:104(3):240-3.

33. Glowacki K, Duncan MJ, Gainforth H, Faulkner G. Barriers and facilitators to physical activity and exercise among adults with depression: a scoping review. Mental Health Physical Activity. 2017;13:108-19.

34. Weatherson KA, Gainforth $H L$, Jung ME. A theoretical analysis of the barriers and facilitators to the implementation of school-based physical activity policies in Canada: a mixed methods scoping review. Implement Sci. 2017;12(1):41.

35. Tricco AC, Lillie E, Zarin W, O'Brien KK, Colquhoun H, Levac D, et al. PRISMA extension for scoping reviews (PRISMA-SCR): checklist and explanation. Ann Intern Med. 2018;169(7):467-73.

36. Cane J, O'Connor D, Michie S. Validation of the theoretical domains framework for use in behaviour change and implementation research. Implement Sci. 2012;7:37.

37. De Cuir-Gunby JT, Marshall PL, McCulloch AW. Developing and using a codebook for the analysis of interview data: an example from a professional development research project. Field Methods. 2011:23(2):136-55.

38. Braun V, Clarke V. Using thematic analysis in psychology. Qual Res Psychol. 2006:3(2):77-101.

39. Pluye P, Gagnon MP, Griffiths F, Johnson-Lafleur J. A scoring system for appraising mixed methods research, and concomitantly appraising qualitative, quantitative and mixed methods primary studies in Mixed Studies Reviews. Int J Nurs Stud. 2009:46(4):529-46.

40. Hong QN, Pluye P, Fabregues S, Bartlett G, Boardman F, Cargo M, et al. Mixed Methods Appraisal Tool (MMAT), version 2018. Canadian Intellectual Property Office, Industry Canada. Registration of Copyright (\#1148552). 
41. Pace R, Pluye P, Bartlett G, Macaulay AC, Salsberg J, Jagosh J, et al. Testing the reliability and efficiency of the pilot mixed methods appraisal tool (MMAT) for systematic mixed studies review. Int J Nurs Stud. 2012;49(1):47-53.

42. Tricco AC, Antony J, Zarin W, Strifler L, Ghassemi M, Ivory J, et al. A scoping review of rapid review methods. BMC Med. 2015;13:224.

43. Abrami PC, Borokhovski E, Bernard RM, Wade CA, Tamim R, Persson T, et al. Issues in conducting and disseminating brief reviews of evidence. Evidence \& Policy. 2010;6(3):371-89.

\section{Publisher's Note}

Springer Nature remains neutral with regard to jurisdictional claims in published maps and institutional affiliations.

Ready to submit your research? Choose BMC and benefit from:

- fast, convenient online submission

- thorough peer review by experienced researchers in your field

- rapid publication on acceptance

- support for research data, including large and complex data types

- gold Open Access which fosters wider collaboration and increased citations

- maximum visibility for your research: over $100 \mathrm{M}$ website views per year

At $\mathrm{BMC}$, research is always in progress.

Learn more biomedcentral.com/submissions 